\title{
On the analytic solutions of the functional equations
}

$$
w_{1} f\left(a_{1} z\right)+w_{2} f\left(a_{2} z\right)+\ldots+w_{n} f\left(a_{n} z\right)=0
$$

\section{Juan Matías Sepulcre and Tomás Vidal}

\begin{abstract}
In this paper it is showed that, given an integer number $n \geq 2$, each zero of an exponential polynomial of the form $w_{1} a_{1}^{z}+$ $w_{2} a_{2}^{z}+\ldots+w_{n} a_{n}^{z}$, with non-null complex numbers $w_{1}, w_{2}, \ldots, w_{n}$ and $a_{1}, a_{2}, \ldots, a_{n}$, produces analytic solutions of the functional equation $w_{1} f\left(a_{1} z\right)+w_{2} f\left(a_{2} z\right)+\ldots+w_{n} f\left(a_{n} z\right)=0$ on certain domains of $\mathbb{C}$, which represents an extension of some existing results in the literature on this functional equation for the case of positive coefficients $a_{j}$ and $w_{j}$.
\end{abstract}

Mathematics Subject Classification (2010). Primary 30Axx; Secondary 39Bxx, 30D05.

Keywords. Functional Equations, Complex Variable, Exponential Polynomials.

\section{Introduction}

The motivation of this paper arises from the study of the functional equation

$$
f(x)+f(2 x)+\ldots+f(n x)=0, x>0, n \in \mathbb{N}, n \geq 2,
$$

introduced in the literature by Mora, Cherruault and Ziadi in 1999 [3], and used for modeling certain processes related to combustion of hydrogen in a car engine for small values of $n[4]$.

From a theoretical point of view, this study is focused on the search of basic solutions of the functional equation

$$
f(z)+f(2 z)+\ldots+f(n z)=0, n \in \mathbb{N}, n \geq 2 .
$$

Preprint 
on the complex domain $\Omega=\mathbb{C} \backslash(-\infty, 0]$. As these solutions are analytic on $\Omega$, the real and imaginary part of their restrictions on $(0, \infty)$ form a family of continuous solutions of functional equation (1.1).

In this sense, there exists a strong connection $[4,7,8]$ between the solutions of (1.1) (or (1.2)) and the zeros of the partial sums of the Riemann zeta function defined as

$$
\zeta_{n}(z):=1+\frac{1}{2^{z}}+\ldots+\frac{1}{n^{z}}, n \in \mathbb{N}, n \geq 2 .
$$

Some of the most relevant properties of these exponential functions are shown in $[4,5,6]$.

On the other hand, in a recent paper [1], the authors study the more general equation

$$
f(x)+f\left(a_{1} x\right)+\ldots+f\left(a_{N} x\right)=0, x>0,
$$

where $0<a_{1}<a_{2}<\ldots<a_{N}$ are positive real numbers and $N \in \mathbb{N}$ with $N \geq 2$. For example, they prove that the set of continuous solutions of (1.3) is an infinite dimensional vector space [1, Theorem 3.1].

In this paper, from the process used for functional equations (1.1) and (1.2), we will firstly construct explicit solutions for equation (1.3) and the more general cases

$$
w_{1} f\left(a_{1} x\right)+w_{2} f\left(a_{2} x\right)+\ldots+w_{n} f\left(a_{n} x\right)=0, x>0,
$$

and

$$
w_{1} f\left(a_{1} z\right)+w_{2} f\left(a_{2} z\right)+\ldots+w_{n} f\left(a_{n} z\right)=0, z \in \mathbb{C} \backslash(-\infty, 0],
$$

where the $a_{j}$ 's are positive real numbers and the $w_{j}$ 's are non-null complex numbers. Thus the functions $\zeta_{n}(z)$, that determine continuous solutions for the case (1.1), will be replaced by other generic exponential polynomials whose zeros will provide a vector space of basic solutions of the functional equations above.

Secondly, we will extend this process to the most general case where the $a_{j}$ 's are non-null complex numbers in order to find analytic solutions of the functional equation on certain domains of $\mathbb{C}$.

Thirdly, we will define a binary relation on the functional equations above which will determine equivalence classes verifying the following property: two equivalent functional equations will have the same solutions and they will be associated to two exponential polynomials with the same set of zeros.

\section{A first generalization}

Let $0<a_{1}<a_{2}<\ldots<a_{n}$ be $n \geq 2$ positive real numbers and $w_{j} \in \mathbb{C} \backslash\{0\}$, $j=1,2, \ldots, n$. In this section we are going to focus on the functional equation

$$
w_{1} f\left(a_{1} x\right)+w_{2} f\left(a_{2} x\right)+\ldots+w_{n} f\left(a_{n} x\right)=0, x>0,
$$


which, in the complex plane, can be written as

$$
w_{1} f\left(a_{1} z\right)+w_{2} f\left(a_{2} z\right)+\ldots+w_{n} f\left(a_{n} z\right)=0 .
$$

Let $F(z)$ be a solution of (2.2) on some complex domain containing the positive real axis, then $g(x):=F(x+i 0), x>0$, is a solution of functional equation (2.1) and the functions of the form

$$
c \operatorname{Re} g(x)+d \operatorname{Im} g(x)
$$

are real solutions of (2.1) for arbitrary real numbers $c, d$. Conversely, if $f(x)$ is a solution of (2.1), then the functions of the form $F(z):=\lambda f(x)+\beta i f(y)$, with $z=x+i y, x, y>0$, and $\lambda, \beta \in \mathbb{C}$, are complex solutions of equation $(2.2)$.

Therefore, in order to find a vector space of basic solutions of (2.2), consider the continuous homomorphism $\varphi(z):=z^{\alpha}$ with $\alpha \in \mathbb{C}$. Thus it must satisfy

$$
w_{1} \varphi\left(a_{1} z\right)+w_{2} \varphi\left(a_{2} z\right)+\ldots+w_{n} \varphi\left(a_{n} z\right)=0
$$

i.e.

$$
w_{1} a_{1}^{\alpha} z^{\alpha}+w_{2} a_{2}^{\alpha} z^{\alpha}+\ldots+w_{n} a_{n}^{\alpha} z^{\alpha}=0
$$

or, equivalently,

$$
z^{\alpha}\left[w_{1} a_{1}^{\alpha}+w_{2} a_{2}^{\alpha}+\ldots+w_{n} a_{n}^{\alpha}\right]=0 .
$$

Hence, $\varphi(z):=z^{\alpha}$ is a solution of (2.2) when $\alpha$ is a zero of the exponential polynomial $P_{n}(z)$ of real frequencies $\log a_{1}, \log a_{2}, \ldots, \log a_{n}$ and complex coefficients $w_{1}, w_{2}, \ldots, w_{n}$, that is $P_{n}(\alpha)=0$ where

$$
P_{n}(z):=w_{1} a_{1}^{z}+w_{2} a_{2}^{z}+\ldots+w_{n} a_{n}^{z} .
$$

Consequently, given $\alpha_{n, j}$ a zero of $P_{n}(z)$, the power functions

$$
f_{n, j}(z):=z^{\alpha_{n, j}}=e^{\alpha_{n, j} \log z}
$$

where $\log z$ is the principal branch of the logarithm, are the key of the existence of solutions of our functional equations. Observe that the functions $f_{n, j}(z)=e^{\alpha_{n, j} \log z}$ are defined on the domain $\Omega:=\mathbb{C} \backslash(-\infty, 0]$.

Particularly, for $z=x>0$, a solution of $(2.1)$ is given by $f_{n, j}(x):=$ $x^{\alpha_{n, j}}$, where $\alpha_{n, j}$ is a zero of the exponential polynomial (2.3). Therefore, if $\alpha_{n, j}=a_{n, j}+i b_{n, j}$, then

$$
f_{n, j}(x)=x^{a_{n, j}+i b_{n, j}}=x^{a_{n, j}} e^{i b_{n, j} \log x},
$$

and we obtain real solutions of (2.1) of the form

$$
x^{a_{n, j}}\left(c \cos \left(b_{n, j} \log x\right)+d \sin \left(b_{n, j} \log x\right)\right), c, d \in \mathbb{R} .
$$

Some results concerning the distribution of the zeros of the exponential polynomials $P_{n}(z)$ of the form $(2.3)$ can be seen in [6]. For example, it was shown that there exist two real numbers $x_{1}, x_{2}$ such that all the zeros of $P_{n}(z)$ are in the strip $\left\{z: x_{1}<\operatorname{Re} z<x_{2}\right\}[6$, Lemma 5].

Now, we will formalize this process through the vector space $\mathcal{A}(\Omega)$ of the analytic functions defined on $\Omega=\mathbb{C} \backslash(-\infty, 0]$ and the vector subspace $\mathcal{V}_{n}, n \geq 2$, of the analytic solutions defined on $\Omega$ of functional equations (2.2). 
We firstly show that $\mathcal{V}_{n}$ is invariant under a certain operator on the space $\mathcal{A}(\Omega)$.

Lemma 1. In the space $\mathcal{A}(\Omega)$, the subspace $\mathcal{V}_{n}, n \geq 2$, is invariant under the linear operator $\mathcal{D}$ defined by $\mathcal{D}(f):=z f^{\prime}$, where $f^{\prime}$ is the derivative of $f$.

Proof. Let $f$ be a function of $\mathcal{V}_{n}$ and let $g$ denote the function $\mathcal{D}(f)$. Then

$$
\begin{gathered}
w_{1} g\left(a_{1} z\right)+w_{2} g\left(a_{2} z\right)+\ldots+w_{n} g\left(a_{n} z\right)= \\
w_{1} a_{1} z f^{\prime}\left(a_{1} z\right)+w_{2} a_{2} z f^{\prime}\left(a_{2} z\right)+\ldots+w_{n} a_{n} z f^{\prime}\left(a_{n} z\right)= \\
z\left(w_{1} f\left(a_{1} z\right)+w_{2} f\left(a_{2} z\right)+\ldots+w_{n} f\left(a_{n} z\right)\right)^{\prime}=0 .
\end{gathered}
$$

Therefore $g \in \mathcal{V}_{n}$ and, consequently, $\mathcal{D}\left(\mathcal{V}_{n}\right) \subset \mathcal{V}_{n}$.

Theorem 2. Let $0<a_{1}<a_{2}<\ldots<a_{n}$ be $n \geq 2$ positive real numbers and $w_{j} \in \mathbb{C} \backslash\{0\}, j=1,2, \ldots, n$. The proper vectors of the operator $\mathcal{D}$ on the space $\mathcal{A}(\Omega)$ are solutions of functional equation (2.2) if and only if the corresponding proper values are the zeros of exponential polynomial (2.3).

Proof. Let $f$ be a proper vector of $\mathcal{D}$ corresponding to a generic proper value $\lambda$. Thus $\mathcal{D}(f)=\lambda f$ and $f$ satisfies the equation

$$
z f^{\prime}=\lambda f
$$

whose solution is

$$
f(z)=K z^{\lambda}
$$

with $K$ an arbitrary constant. Now, the expression

$$
w_{1} f\left(a_{1} z\right)+w_{2} f\left(a_{2} z\right)+\ldots+w_{n} f\left(a_{n} z\right)=K z^{\lambda}\left(w_{1} a_{1}^{\lambda}+w_{2} a_{2}^{\lambda}+\ldots+w_{n} a_{n}^{\lambda}\right)
$$

vanishes for any $z \in \Omega$ if and only if

$$
w_{1} a_{1}^{\lambda}+w_{2} a_{2}^{\lambda}+\ldots+w_{n} a_{n}^{\lambda}=0,
$$

that is, if and only if $\lambda$ is a zero of $P_{n}(z)=w_{1} e^{z \log a_{1}}+w_{2} e^{z \log a_{2}}+\ldots+$ $w_{n} e^{z \log a_{n}}$. Hence, the theorem follows.

Consequently, from the preceding results, we obtain the following important results.

Corollary 3. Let $0<a_{1}<a_{2}<\ldots<a_{n}$ be $n \geq 2$ positive real numbers and $w_{j} \in \mathbb{C} \backslash\{0\}, j=1,2, \ldots, n$. For each $n \geq 2$, the functions $f_{n, j}(z)=$ $e^{\alpha_{n, j} \log z}$, with $\alpha_{n, j}$ belonging to the set of the zeros of $P_{n}(z)$, defined in (2.3), are analytic solutions of functional equation (2.2) on $\Omega$.

Corollary 4. Fix an integer number $n \geq 2$, let $0<a_{1}<a_{2}<\ldots<a_{n}$ be positive real numbers and $w_{j} \in \mathbb{C} \backslash\{0\}, j=1,2, \ldots, n$. Thus every zero of the exponential polynomial $P_{n}(z)=w_{1} e^{z \log a_{1}}+w_{2} e^{z \log a_{2}}+\ldots+w_{n} e^{z \log a_{n}}$ generates a vector space of real continuous solutions of functional equation (2.1). 
Particularly, given a zero of $P(z)=1+e^{z \log a_{1}}+e^{z \log a_{2}}+\ldots+e^{z \log a_{n}}$, the family of functions on the interval $(0, \infty)$ of the form

$$
\left\{c \operatorname{Re}\left(x^{\alpha_{n, j}}\right)+d \operatorname{Im}\left(x^{\alpha_{n, j}}\right): c, d \in \mathbb{R}\right\}
$$

is a vector space of continuous solutions of functional equation (1.3) studied in [1].

Furthermore, an easy consequence of the previous corollary is that every real continuous solution $f(x)$ of $(2.1)$ defines a function

$$
h(x)= \begin{cases}f(x), & \text { if } x>0 \\ 0, & \text { if } x=0 \\ f(-x), & \text { if } x<0\end{cases}
$$

which is a continuous solution, except possibly at the point 0 , of the functional equation

$$
w_{1} f\left(a_{1} x\right)+w_{2} f\left(a_{2} x\right)+\ldots+w_{n} f\left(a_{n} x\right)=0, x \in \mathbb{R} .
$$

Finally, for the particular case that the $w_{j}$ 's are positive real numbers, we shall show that any solution of functional equation (2.2) cannot be analytic at the origin, except the trivial solution $f \equiv 0$, and the functions $f_{n, j}(z)=$ $z^{\alpha_{n, j}}$ with $\alpha_{n, j}$ belonging to the set of zeros of $P_{n}(z)$ are linearly independent in the vector space $\mathcal{A}(\Omega)$.

Proposition 5. Let $0<a_{1}<a_{2}<\ldots<a_{n}$ be $n \geq 2$ positive real numbers and $w_{j}>0, j=1,2, \ldots, n$. Let $f(z)$ be any non-trivial complex solution of functional equation (2.2). Then $f(z)$ is not analytic at 0 .

Proof. Assume that there exists a solution $f(z)$ of $(2.2)$ which is analytic at 0 and, therefore, analytic on an open set $U$ containing 0 . Hence, by considering the derivatives of all orders of $f(z)$ on $U$, we have

$$
w_{1} a_{1}^{m} f^{(m)}\left(a_{1} z\right)+w_{2} a_{2}^{m} f^{(m)}\left(a_{2} z\right)+\ldots+w_{n} a_{n}^{m} f^{(m)}\left(a_{n} z\right)=0
$$

for each non-negative integer $m$. In particular, for $z=0$ we deduce that

$$
\left(w_{1} a_{1}^{m}+w_{2} a_{2}^{m}+\ldots+w_{n} a_{n}^{m}\right) f^{(m)}(0)=0
$$

and, therefore, $f^{(m)}(0)=0$ for all integer $m \geq 0$. Consequently, $f \equiv 0$ and the proposition follows.

Proposition 6. Let $0<a_{1}<a_{2}<\ldots<a_{n}$ be $n \geq 2$ positive real numbers and $w_{j}>0, j=1,2, \ldots, n$. Then the analytic solutions $f_{n, j}(z)=z^{\alpha_{n, j}}$ of (2.2), with $\alpha_{n, j}$ belonging to the set of zeros of $P_{n}(z)=w_{1} e^{z \log a_{1}}+w_{2} e^{z \log a_{2}}+$ $\ldots+w_{n} e^{z \log a_{n}}$, are linearly independent in the vector space $\mathcal{A}(\Omega)$.

Proof. From Corollary 3, observe that the functions $f_{n, j}(z)=z^{\alpha_{n, j}}$ with $\alpha_{n, j}$ a zero of $P_{n}(z)$, are analytic solutions of functional equation (2.2) on $\Omega$. Let $\alpha_{n, 1}, \alpha_{n, 2}, \ldots, \alpha_{n, m}$ be $m$ distinct zeros of $P_{n}(z)$ with $\alpha_{n, j}=a_{n, j}+i b_{n, j}$, $j=1,2, \ldots, m$. Observe that, since $P_{n}(x) \neq 0$ for all $x \in \mathbb{R}$, then $b_{n}:=$ $\max \left\{\left|b_{n, j}\right|: j=1,2, \ldots, m\right\}>0$. Also, we can determine $t_{n}>1$ such that $b_{n} \log t_{n}<\pi$. Then, we have that $-\pi<b_{n, j} \log t_{n}<\pi, j=1,2, \ldots, m$, and therefore the principal argument of $t_{n}^{\alpha_{n, j}}$ is $b_{n, j} \log t_{n}$ for each $j=1,2, \ldots, m$. 
Now, consider the numbers $c_{j}:=t_{n}^{j-1}, j=1,2, \ldots, m$, and let $g_{1}, g_{2}, \ldots, g_{m}$ denote the power functions $z^{\alpha_{n, 1}}, z^{\alpha_{n, 2}}, \ldots, z^{\alpha_{n, m}}$ respectively. Thus the determinant

$$
D=\left|\begin{array}{cccc}
g_{1}\left(c_{1}\right) & g_{2}\left(c_{1}\right) & \cdots & g_{m}\left(c_{1}\right) \\
g_{1}\left(c_{2}\right) & g_{2}\left(c_{2}\right) & \cdots & g_{m}\left(c_{2}\right) \\
\vdots & \vdots & \ddots & \vdots \\
g_{1}\left(c_{m}\right) & g_{2}\left(c_{m}\right) & \cdots & g_{m}\left(c_{m}\right)
\end{array}\right|=\left|\begin{array}{cccc}
1 & 1 & \cdots & 1 \\
d_{1} & d_{2} & \cdots & d_{m} \\
\vdots & \vdots & \ddots & \vdots \\
d_{1}^{m-1} & d_{2}^{m-1} & \cdots & d_{m}^{m-1}
\end{array}\right|
$$

where $d_{j}:=t_{n}^{\alpha_{n, j}}, j=1,2, \ldots, m$, is of Vandermonde type and so

$$
D=\prod_{k>j(k, j=1, \ldots, m)}\left(d_{k}-d_{j}\right) .
$$

Finally, since the $d_{j}$ 's are distinct because they have different arguments or absolute values, we deduce that $D \neq 0$ and the functions $f_{n, j}(z)$ are linearly independent.

In particular, we deduce that the set of continuous solutions of particular case (1.3) is an infinite dimensional vector space, that was also proved in [1, Theorem 3.1].

\section{More general cases}

In this section we will handle the more general functional equation

$$
w_{1} f\left(a_{1} z\right)+w_{2} f\left(a_{2} z\right)+\ldots+w_{n} f\left(a_{n} z\right)=0, z \in \mathbb{C},
$$

where the $w_{j}$ 's and also the $a_{j}$ 's are non-null complex numbers and $n \geq 2$ is an integer number. Our first purpose is to extend the result of Corollary 3 to this equation.

In this manner, initially, by considering the principal branch of the logarithm function, denoted by $\log z$, some explicit analytic solutions of (3.1) on a certain domain included in $\mathbb{C} \backslash(-\infty, 0]$ will be generated by the exponential polynomials

$$
Q_{n}(z)=w_{1} a_{1}^{z}+w_{2} a_{2}^{z}+\ldots+w_{n} a_{n}^{z} .
$$

Observe that if $a_{j}>0, j=1,2, \ldots, n$, (the case of functional equation $(2.2))$ and $z \in \mathbb{C} \backslash(-\infty, 0]$, then $a_{j} z \in \mathbb{C} \backslash(-\infty, 0]$. Now, if the $a_{j}$ 's are nonnull complex numbers (not necessarily positive real numbers), this condition is not assured and we must take neighborhoods of a given point in order to find analytic solutions of $(3.1)$ on a certain domain included in $\mathbb{C} \backslash(-\infty, 0]$.

Theorem 7. Let $n \geq 2$ be an integer number and $a_{j}, w_{j} \in \mathbb{C} \backslash\{0\}, j=$ $1,2, \ldots, n$. Thus for each $z_{0} \in \Omega$ such that $a_{j} z_{0} \notin(-\infty, 0]$ for $j=1,2, \ldots, n$, the functions $f_{n, j}(z):=e^{\alpha_{n, j} \log z}$, with $\alpha_{n, j}$ belonging to the set of the zeros of $Q_{n}(z)$, defined in (3.2), are analytic solutions of (3.1) on a certain neighborhood of $z_{0}$. 
Proof. Let $R_{0}^{-}$denote the set $(-\infty, 0]$. Fix $z_{0} \in \Omega=\mathbb{C} \backslash R_{0}^{-}$such that $a_{j} z_{0} \notin R_{0}^{-}$and define $M:=\max \left\{\left|a_{1}\right|,\left|a_{2}\right|, \ldots,\left|a_{n}\right|\right\}>0$ and

$$
d:=\min \left\{d\left(z_{0}, R_{0}^{-}\right), d\left(a_{1} z_{0}, R_{0}^{-}\right), d\left(a_{2} z_{0}, R_{0}^{-}\right), \ldots, d\left(a_{n} z_{0}, R_{0}^{-}\right)\right\}>0,
$$

where $d\left(\cdot, R_{0}^{-}\right)$is the distance function from a point to the set $R_{0}^{-}$. Thus, by taking $\varepsilon:=\min \left\{d, \frac{d}{M}\right\}>0$, if $z \in D\left(z_{0}, \varepsilon\right):=\left\{z \in \mathbb{C}:\left|z-z_{0}\right|<\varepsilon\right\}$ we have $\left|z-z_{0}\right|<\varepsilon \leq d$ and $\left|a_{j} z-a_{j} z_{0}\right|<a_{j} \varepsilon \leq M \varepsilon \leq d$ for $j=1,2, \ldots, n$. Hence $D\left(z_{0}, \varepsilon\right) \subset \Omega$.

Finally, if $f_{n, j}(z)=e^{\alpha_{n, j} \log z}$, where $\alpha_{n, j}$ is a zero of $Q_{n}(z)$, and $z \in$ $D\left(z_{0}, \varepsilon\right)$, then

$$
\begin{gathered}
w_{1} f_{n, j}\left(a_{1} z\right)+w_{2} f_{n, j}\left(a_{2} z\right)+\ldots+w_{n} f_{n, j}\left(a_{n} z\right)= \\
w_{1}\left(a_{1} z\right)^{\alpha_{n, j}}+w_{2}\left(a_{2} z\right)^{\alpha_{n, j}}+\ldots+w_{n}\left(a_{n} z\right)^{\alpha_{n, j}}= \\
z^{\alpha_{n, j}}\left[w_{1} a_{1}^{\alpha_{n, j}}+w_{2} a_{2}^{\alpha_{n, j}}+\ldots+w_{n} a_{n}^{\alpha_{n, j}}\right]= \\
z^{\alpha_{n, j}} Q_{n}\left(\alpha_{n, j}\right)=0
\end{gathered}
$$

and the result follows.

Observe that the theorem above requires that $z_{0} \in \Omega$ with $a_{j} z_{0} \notin$ $(-\infty, 0]$ for $j=1,2, \ldots, n$, Now, we will generalize this result by obtaining analytic solutions of (2.3) on certain neighborhoods of any point $z_{0} \neq 0$. So, given $\theta \in \mathbb{R}$, let $\log _{\theta} z$ denote the branch of the logarithm function such that the argument of $z$ is in $[\theta, \theta+2 \pi)$, that is $\log _{\theta} z=\log |z|+i \arg _{\theta} z$, with $\arg _{\theta}$ the unique argument of $z$ in $[\theta, \theta+2 \pi)$. Recall that the function $\log _{\theta} z$ is analytic on $\mathbb{C} \backslash R_{\theta}$, where $R_{\theta}$ is the ray $\left\{r e^{i \theta}: r \geq 0\right\}$.

Theorem 8. Let $n \geq 2$ be an integer number and $a_{j}, w_{j} \in \mathbb{C} \backslash\{0\}, j=$ $1,2, \ldots, n$. Thus for each non-null complex number $z_{0}$, there exists a real number $\theta_{0}$ such that the functions $f_{n, j}(z)=e^{\alpha_{n, j} \log _{\theta_{0}} z}$, with $\alpha_{n, j}$ belonging to the set of the zeros of $Q_{n}(z)$, defined in (3.2), are analytic solutions of (3.1) on a certain neighborhood of $z_{0}$.

Proof. Fix $z_{0} \in \mathbb{C} \backslash\{0\}$ and choose $\theta_{0} \in[-\pi, \pi)$ such that $\theta_{0} \neq \operatorname{Arg}\left(z_{0}\right)$ and $\theta_{0} \neq \operatorname{Arg}\left(a_{j} z_{0}\right)$ for $j=1,2, \ldots, n$. Now, by following the proof of Theorem 7, we will demonstrate that there exists $\varepsilon>0$ such that $D\left(z_{0}, \varepsilon\right) \subset \mathbb{C} \backslash R_{\theta_{0}}$. Indeed, let $M:=\max \left\{\left|a_{1}\right|,\left|a_{2}\right|, \ldots,\left|a_{n}\right|\right\}>0$ and

$$
d:=\min \left\{d\left(z_{0}, R_{\theta_{0}}\right), d\left(a_{1} z_{0}, R_{\theta_{0}}\right), d\left(a_{2} z_{0}, R_{\theta_{0}}\right), \ldots, d\left(a_{n} z_{0}, R_{\theta_{0}}\right)\right\}>0
$$

and take $\varepsilon:=\min \left\{d, \frac{d}{M}\right\}>0$, then if $z \in D\left(z_{0}, \varepsilon\right)$ we have $\left|z-z_{0}\right|<\varepsilon \leq d$ and $\left|a_{j} z-a_{j} z_{0}\right|<a_{j} \varepsilon \leq M \varepsilon \leq d$ and the result follows.

Finally, if $f_{n, j}(z)=e^{\alpha_{n, j} \log _{\theta_{0}} z}$, with $\alpha_{n, j}$ belonging to the set of the zeros of $Q_{n}(z)$, and $z \in D\left(z_{0}, \varepsilon\right)$ then

$$
\begin{gathered}
w_{1} f_{n, j}\left(a_{1} z\right)+w_{2} f_{n, j}\left(a_{2} z\right)+\ldots+w_{n} f_{n, j}\left(a_{n} z\right)= \\
w_{1} e^{\alpha_{n, j} \log _{\theta_{0}}\left(a_{1} z\right)}+w_{2} e^{\alpha_{n, j} \log _{\theta_{0}}\left(a_{2} z\right)}+\ldots+w_{n} e^{\alpha_{n, j} \log _{\theta_{0}}\left(a_{n} z\right)}= \\
e^{\alpha_{n, j} \log _{\theta_{0}} z}\left[w_{1} e^{\alpha_{n, j} \log _{\theta_{0}} a_{1}}+w_{2} e^{\alpha_{n, j} \log _{\theta_{0}} a_{2}}+\ldots+w_{n} e^{\alpha_{n, j} \log _{\theta_{0}} a_{n}}\right]= \\
z^{\alpha_{n, j}} Q_{n}\left(\alpha_{n, j}\right)=0
\end{gathered}
$$


and, therefore, the functions $f_{n, j}(z)$ are analytic solutions of $(3.1)$ on $D\left(z_{0}, \varepsilon\right)$.

In order to extend this process, let $P: \mathbb{C} \backslash\{0\} \times \mathbb{C} \backslash\{0\} \longmapsto \mathbb{R}^{+} \times \mathbb{R}$ denote the application defined as

$$
P\left(z_{1}, z_{2}\right)=P\left(r_{1} e^{i \beta_{1}}, r_{2} e^{i \beta_{2}}\right):=\left(r_{1} r_{2}, \beta_{1}+\beta_{2}\right),
$$

where $z_{1}$ and $z_{2}$ are non-null complex numbers expressed in the form $z_{j}=$ $r_{j} e^{i \beta_{j}}$ with $r_{j}>0$ and $\beta_{j}=\arg _{-\pi} z_{j} \in[-\pi, \pi), j=1,2$. So, now we consider the functional equation

$$
w_{1} f\left(P\left(a_{1}, z\right)\right)+w_{2} f\left(P\left(a_{2}, z\right)\right)+\ldots+w_{n} f\left(P\left(a_{n}, z\right)\right)=0, z \in \mathbb{C} \backslash\{0\},
$$

where the $w_{j}$ 's and the $a_{j}$ 's are non-null complex numbers and $f$ is an application of the form $f: \mathbb{R}^{+} \times \mathbb{R} \rightarrow \mathbb{C}$.

We will also use the function $\log ^{*}: \mathbb{R}^{+} \times \mathbb{R} \rightarrow \mathbb{C}$ defined as $\log ^{*}(r, \beta):=$ $\log r+i \beta$.

Theorem 9. Let $n \geq 2$ be an integer number and $a_{j}, w_{j} \in \mathbb{C} \backslash\{0\}, j=$ $1,2, \ldots, n$. Thus the functions $f_{n, j}: \mathbb{R}^{+} \times \mathbb{R} \rightarrow \mathbb{C}$ defined as $f_{n, j}(r, \beta):=$ $e^{\alpha_{n, j} \log ^{*}(r, \beta)}$, with $\alpha_{n, j}$ belonging to the set of the zeros of the exponential polynomial $Q_{n}(z)$, defined in (3.2), are continuous solutions of functional equation (3.3) on all the domain $\mathbb{C} \backslash\{0\}$.

Proof. Let $a_{j}=\left|a_{j}\right| e^{i \beta_{j}}$, where $\beta_{j}=\arg _{-\pi} a_{j}$ is the principal argument of $a_{j}, j=1,2, \ldots, n$. Consider $f_{n, j}(r, \beta)=e^{\alpha_{n} \log ^{*}(r, \beta)}$, where $\alpha_{n, j}$ is a zero of $Q_{n}(z)$ and take $z=r e^{i \beta} \in \mathbb{C} \backslash\{0\}$ with $\beta=\arg _{-\pi} z$, then

$$
\begin{gathered}
w_{1} f_{n, j}\left(P\left(a_{1}, z\right)\right)+w_{2} f_{n, j}\left(P\left(a_{2}, z\right)\right)+\ldots+w_{n} f_{n, j}\left(P\left(a_{n}, z\right)\right)= \\
w_{1} e^{\alpha_{n, j} \log ^{*}\left(\left|a_{1}\right| r, \beta_{1}+\beta\right)}+w_{2} e^{\alpha_{n, j} \log ^{*}\left(\left|a_{2}\right| r, \beta_{2}+\beta\right)}+\ldots+ \\
w_{n} e^{\alpha_{n, j} \log ^{*}\left(\left|a_{n}\right| r, \beta_{n}+\beta\right)}= \\
w_{1} e^{\alpha_{n, j}\left[\log \left(\left|a_{1}\right| r\right)+i\left(\beta_{1}+\beta\right)\right]}+w_{2} e^{\alpha_{n, j}\left[\log \left(\left|a_{2}\right| r\right)+i\left(\beta_{2}+\beta\right)\right]}+\ldots+ \\
w_{n} e^{\alpha_{n, j}\left[\log \left(\left|a_{n}\right| r\right)+i\left(\beta_{n}+\beta\right)\right]}= \\
e^{\alpha_{n, j}(\log r+i \beta)}\left[w_{1} e^{\alpha_{n, j}\left(\log \left|a_{1}\right|+i \beta_{1}\right)}+w_{2} e^{\alpha_{n, j}\left(\log \left|a_{2}\right|+i \beta_{2}\right)}+\ldots+\right. \\
\left.w_{n} e^{\alpha_{n, j}\left(\log \left|a_{n}\right|+i \beta_{n}\right)}\right]= \\
e^{\alpha_{n, j}(\log r+i \beta)}\left[w_{1} e^{\alpha_{n, j} \log a_{1}}+w_{2} e^{\alpha_{n, j} \log a_{2}}+\ldots+\right. \\
e^{\alpha_{n, j}(\log r+i \beta)} Q_{n}\left(\alpha_{n, j}\right)=0
\end{gathered}
$$

and the theorem follows.

\section{Equivalence classes of functional equations}

Now we will define a binary relation on the class of generic functional equations of the form (3.1). 
Definition 10. For each integer number $n \geq 2$, let $\mathcal{F}_{n}$ be the set of all functional equations of the form (3.1) with $n$ terms. We will say that two functionals equations in $\mathcal{F}_{n}$,

$$
w_{1} f\left(a_{1} z\right)+w_{2} f\left(a_{2} z\right)+\ldots+w_{n} f\left(a_{n} z\right)=0, z \in \mathbb{C},
$$

and

$$
v_{1} f\left(b_{1} z\right)+v_{2} f\left(b_{2} z\right)+\ldots+v_{n} f\left(b_{n} z\right)=0, z \in \mathbb{C},
$$

are equivalent when there exist $\gamma \in \mathbb{C}$ and $c \in \mathbb{C} \backslash\{0\}$ such that $v_{j}=c w_{j}$ and $b_{j}=a_{j} e^{\gamma}$ for each $j=1,2, \ldots, n$.

Note that the binary relation defined above on the class $\mathcal{F}_{n}$ is clearly an equivalence relation.

We showed in Theorems 7 or 8 that the zeros of the exponential polynomials $Q_{n}(z)$ of the form (3.2) provide solutions of functional equation (3.1) on certain domains of the complex plane. Now we will prove that two exponential polynomials which are associated to two equivalent functional equations have the same set of zeros.

In this sense, given $Q_{n}(z)$ an exponential polynomial of the form (3.2) let $Z\left(Q_{n}\right)$ denote the set $\left\{z \in \mathbb{C}: Q_{n}(z)=0\right\}$ of the zeros of $Q_{n}(z)$.

Proposition 11. For each $n \geq 2$, two equivalent functional equations in $\mathcal{F}_{n}$ have associated two exponential polynomials with the same set of zeros.

Proof. Let

$$
w_{1} f\left(a_{1} z\right)+w_{2} f\left(a_{2} z\right)+\ldots+w_{n} f\left(a_{n} z\right)=0, z \in \mathbb{C}
$$

and

$$
v_{1} f\left(b_{1} z\right)+v_{2} f\left(b_{2} z\right)+\ldots+v_{n} f\left(b_{n} z\right)=0, z \in \mathbb{C},
$$

be two equivalent functional equations in $\mathcal{F}_{n}$ and $c, \gamma \in \mathbb{C}$, with $c \neq 0$, such that $v_{j}=c w_{j}$ and $b_{j}=a_{j} e^{\gamma}$ for each $j=1,2, \ldots, n$. Thus, from Theorems 7 and 8 , their associated exponential polynomials are

$$
Q(z)=w_{1} a_{1}^{z}+w_{2} a_{2}^{z}+\ldots+w_{n} a_{n}^{z}
$$

and

$$
R(z)=v_{1} b_{1}^{z}+v_{2} b_{2}^{z}+\ldots+v_{n} b_{n}^{z},
$$

respectively. Now, by taking the conditions of the coefficients into account, note that $R(z)=c e^{\gamma z} Q(z)$ and, since $c e^{\gamma z}$ has no zeros, then the sets $Z(Q)$ and $Z(R)$ are the same.

As a corollary, the sets of the analytic solutions of equivalent functional equations, that are generated by the associated exponential polynomials $Q_{n}(z)$ of the form (3.2), are the same.

Corollary 12. Given an integer number $n \geq 2$, the sets of solutions of the form $f_{n, j}(z)$ that appear in Theorems 7 or 8 of two equivalent functional equations in $\mathcal{F}_{n}$ are the same.

The converse of Proposition 11 is also true. 
Proposition 13. Two exponential polynomials of the form (3.2) with the same set of zeros have associated two equivalent functional equations in $\mathcal{F}_{n}$ for some integer number $n \geq 2$.

Proof. Suppose that $Q(z)$ and $R(z)$ are two exponential polynomials so that $Z(Q)=Z(R)=\left\{z_{1}, z_{2}, \ldots, z_{l}, \ldots\right\}$ and their zeros have associated the same multiplicity. Then, by Hadamard factorization theorem [2, Theorem 4.4.3], we have

$$
Q(z)=z^{k} e^{A_{Q} z+B_{Q}} H(z)
$$

and

$$
R(z)=z^{k} e^{A_{R} z+B_{R}} H(z)
$$

with $k$ the order of the zero at $z=0, A_{Q}, A_{R}, B_{Q}, B_{R} \in \mathbb{C}$ and $H(z)=$ $\prod_{l \geq 1} E_{h}\left(\frac{z}{z_{l}}\right)$ for some integer number $h$ depending on the exponent of convergence of $\left\{z_{l}\right\}$, where

$$
E_{h}(z)=\left\{\begin{array}{ll}
1-z & \text { if } h=0 \\
(1-z) \exp \left(z+\frac{z^{2}}{2}+\ldots+\frac{z^{h}}{h}\right) & \text { if } h \geq 1
\end{array} .\right.
$$

Furthermore, from (4.1) and (4.2), we have

$$
Q(z)=\frac{R(z)}{e^{A_{R} z+B_{R}}} e^{A_{Q} z+B_{Q}},
$$

i.e.

$$
Q(z)=e^{\gamma z} c R(z),
$$

where $c=e^{B_{Q}-B_{R}}$ and $\gamma=A_{Q}-A_{R}$. Therefore, if $Q(z)$ is of the form

$$
w_{1} a_{1}^{z}+w_{2} a_{2}^{z}+\ldots+w_{n} a_{n}^{z},
$$

which is associated to the functional equation

$$
w_{1} f\left(a_{1} z\right)+w_{2} f\left(a_{2} z\right)+\ldots+w_{n} f\left(a_{n} z\right)=0, z \in \mathbb{C},
$$

then

$$
R(z)=c w_{1} a_{1}^{z} e^{\gamma z}+c w_{2} a_{2}^{z} e^{\gamma z}+\ldots+c w_{n} a_{n}^{z} e^{\gamma z}
$$

and it is associated to the functional equation

$$
v_{1} f\left(b_{1} z\right)+v_{2} f\left(b_{2} z\right)+\ldots+v_{n} f\left(b_{n} z\right)=0, z \in \mathbb{C},
$$

with $v_{j}=c w_{j}$ and $b_{j}=a_{j} e^{\gamma}$ for each $j=1,2, \ldots, n$. Consequently, functional equations (4.3) and (4.4) are equivalent in $\mathcal{F}_{n}$ and the result follows.

Furthermore, the equivalent functional equations in $\mathcal{F}_{n}$ have the same set of solutions defined on all domain $\mathbb{C}$.

Proposition 14. For each integer number $n \geq 2$, two equivalent functional equations in $\mathcal{F}_{n}$ have the same solutions defined on $\mathbb{C}$. 
Proof. Let

$$
w_{1} f\left(a_{1} z\right)+w_{2} f\left(a_{2} z\right)+\ldots+w_{n} f\left(a_{n} z\right)=0, z \in \mathbb{C},
$$

and

$$
v_{1} f\left(b_{1} z\right)+v_{2} f\left(b_{2} z\right)+\ldots+v_{n} f\left(b_{n} z\right)=0, z \in \mathbb{C},
$$

be two equivalent functional equations in $\mathcal{F}_{n}$ and $c, \gamma \in \mathbb{C}$, with $c \neq 0$, such that $v_{j}=c w_{j}$ and $b_{j}=a_{j} e^{\gamma}$ for each $j=1,2, \ldots, n$. Firstly, let $f_{1}(z)$ be a solution of (4.5), defined on $\mathbb{C}$, then $f_{1}(z)$ satisfies (4.6) if and only if

$$
c w_{1} f_{1}\left(a_{1} e^{\gamma} z\right)+c w_{2} f_{1}\left(a_{2} e^{\gamma} z\right)+\ldots+c w_{n} f_{1}\left(a_{n} e^{\gamma} z\right)=0
$$

which, by replacing $z$ by $\frac{z}{e^{\gamma}}$, is equal to

$$
w_{1} f_{1}\left(a_{1} z\right)+w_{2} f_{1}\left(a_{2} z\right)+\ldots+w_{n} f_{1}\left(a_{n} z\right)=0
$$

which is true. Conversely, let $f_{2}(z)$ be a solution of (4.6), defined on $\mathbb{C}$, then $f_{2}(z)$ satisfies $(4.5)$ if and only if

$$
\frac{v_{1}}{c} f\left(b_{1} e^{-\gamma} z\right)+\frac{v_{2}}{c} f\left(b_{2} e^{-\gamma} z\right)+\ldots+\frac{v_{n}}{c} f\left(b_{n} e^{-\gamma} z\right)=0,
$$

which, by replacing $z$ by $z e^{\gamma}$, is equal to

$$
v_{1} f_{2}\left(b_{1} z\right)+v_{2} f_{2}\left(b_{2} z\right)+\ldots+v_{n} f_{2}\left(b_{n} z\right)=0
$$

which is true.

Given $n \geq 2$, let

$$
w_{1} f\left(a_{1} z\right)+w_{2} f\left(a_{2} z\right)+\ldots+w_{n} f\left(a_{n} z\right)=0, z \in \mathbb{C},
$$

be a functional equation in $\mathcal{F}_{n}$ and $Q_{n}(z)=w_{1} a_{1}^{z}+w_{2} a_{2}^{z}+\ldots+w_{n} a_{n}^{z}$ the exponential polynomial associated to it. As you can see above, the (analytic and non-analytic) solutions defined on $\mathbb{C}$ of equivalent functional equations in $\mathcal{F}_{n}$ are the same. For example, the functions

$$
g_{n, j}(z)=\left\{\begin{array}{ll}
e^{\alpha_{n, j} \log z} & \text { if } z \neq 0 \\
0 & \text { if } z=0
\end{array},\right.
$$

with $\alpha_{n, j}$ belonging to the set of the zeros of $Q_{n}(z)$, are solutions defined on $\mathbb{C}$ of the functional equation above, but they are also solutions of any equivalent functional equation

$$
v_{1} f\left(b_{1} z\right)+v_{2} f\left(b_{2} z\right)+\ldots+v_{n} f\left(b_{n} z\right)=0, z \in \mathbb{C},
$$

with $\gamma \in \mathbb{C}$ and $c \in \mathbb{C} \backslash\{0\}$ such that $v_{j}=c w_{j}$ and $b_{j}=a_{j} e^{\gamma}$ for each $j=1,2, \ldots, n$. 


\section{References}

[1] J.M. Almira and Kh. F. Abu-Helaiel, On solutions of $f(x)+f\left(a_{1} x\right)+\ldots+$ $f\left(a_{N} x\right)=0$ and related equations, Ann. Tiberiu Popoviciu Semin. Funct. Equ. Approx. Convexity. 9 (2011), 3-17.

[2] R. B. Ash, Complex Variables, Academic Press, London, 1971.

[3] G. Mora, Y. Cherruault and A. Ziadi, Functional equations generating spacedensifying curves, Computers and Math. with Appl. 39 (2000), 45-55.

[4] G. Mora, A note on the functional equation $F(z)+F(2 z)+\cdots+F(n z)=0$, J. Math. Anal. Appl. 340 (2008), 466-475.

[5] G. Mora and J.M. Sepulcre, On the distribution of zeros of a sequence of entire functions approaching the Riemann zeta function, J. Math. Anal. Appl. 350 (2009), 409-415.

[6] G. Mora and J.M. Sepulcre, The critical strips of the sums $1+2^{z}+$ $\ldots+N^{z}$, Abstr. Appl. Anal. 2011 (2011), Article ID 909674, 15 pages, doi:10.1155/2011/909674.

[7] G. Mora and J.M. Sepulcre, The zeros of Riemann zeta partial sums yield solutions to $f(x)+f(2 x)+\ldots+f(n x)=0$, Mediterr. J. Math. 10 (2013), 3, 1221-1232.

[8] J.M. Sepulcre, On some solutions of a functional equation related to the partial sums of the Riemann zeta function, Bull. Korean Math. Soc. 51 (2014), 1, $29-41$.

Juan Matías Sepulcre

Department of Mathematical Analysis

University of Alicante

P.O. Box 99

03080 Alicante

Spain

e-mail: JM.Sepulcre@ua.es

Tomás Vidal

University of Alicante

Spain

e-mail: tmvg@alu.ua.es 